\title{
Untersuchungen an Thermoketten
}

\author{
X. Elektrolytschmelzen
}

R. HAASE und J. Richter

Institut für Physikalische Chemie der Rheinisch-Westfälischen Technischen Hochschule Aachen

(Z. Naturforsch. 24 a, 418-423 [1969]; eingegangen am 26. November 1968)

Es werden Thermoketten betrachtet, die zwei Elektrolyte mit insgesamt drei Ionenarten in geschmolzenem Zustand enthalten. Mit Hilfe der Methoden der Thermodynamik der irreversiblen Prozesse lassen sich die allgemeinen Ausdrücke für den Anfangswert und den Endwert der Thermokraft einer solchen Thermokette gewinnen. Die aus den Messungen bestimmbaren charakteristischen Größen sind die beiden Überführungsentropien der den Elektrolyten nicht gemeinsamen Ionenarten, gemessen relativ zur gemeinsamen Ionensorte. Die entsprechenden Formeln für Thermoketten mit reinen flüssigen Elektrolyten lassen sich als Grenzfälle ableiten. Messungen von Sundheim und Kellner an Thermoketten, kombiniert mit anderen Literaturdaten, ergeben die Überführungsentropien der Natrium- und Silberionen für die binäre Schmelze $\mathrm{NaNO}_{3}+\mathrm{AgNO}_{3}$ in Abhängigkeit von der Zusammensetzung bei $310^{\circ} \mathrm{C}$. Anhangweise werden die Konsequenzen eines einfachen Ansatzes für die Überführungszahlen, wie er für $\mathrm{NaNO}_{3}+\mathrm{AgNO}_{3}$ zu gelten scheint, besprochen. Dabei werden auch Konzentrationsketten behandelt.

Während die bisherigen Folgen dieser Reihe sich stets mit Lösungen von Elektrolyten in Wasser ${ }^{1-5}$ oder in einem anderen neutralen Lösungsmittel ${ }^{6}$ befaßten, soll in der vorliegenden Arbeit auf Elektrolytschmelzen eingegangen werden, auf Systeme also, die kein „Lösungsmittel“ enthalten.

Der Einfachheit halber beschränken wir die Diskussion auf binäre Elektrolytschmelzen mit insgesamt drei Ionenarten, d. h. auf Systeme des Typs $\mathrm{NaNO}_{3}+\mathrm{AgNO}_{3}$ oder $\mathrm{PbCl}_{2}+\mathrm{PbBr}_{2}$, weil in neuerer Zeit Thermoketten mit Salzschmelzen dieser Art experimentell untersucht worden sind ${ }^{7-10}$.

\section{Grundlagen}

Wir sprechen bei einer Salzschmelze wie $\mathrm{PbCl}_{2}$ $+\mathrm{PbBr}_{2}$ kurz von den Ionenarten $\mathrm{Pb}^{++}, \mathrm{Cl}^{-}$und $\mathrm{Br}^{-}$. In Wirklichkeit werden oft komplexe Ionen oder undissoziierte Elektrolytmoleküle bzw. Ionenpaare vorliegen. Man kann - mindestens bei der thermodynamisch-phänomenologischen Behandlung der Transporterscheinungen - die Schwierigkeiten

1 R. HaAse, Z. Phys. Chem. Frankfurt 11, 379 [1957]; 13, 21 [1957]; 14, 292 [1958].

2 R. Haase u. H. Schönert, Z. Phys. Chem. Frankfurt 25, 193 [1960].

${ }^{3}$ R. Haase, K. Hoch u. H. Schönert, Z. Phys. Chem. Frankfurt 27, 421 [1961].

${ }^{4}$ R. Haase u. G. Behrend, Z. Phys. Chem. Frankfurt 31, 375 [1962].

5 R. HaAse u. K. Носн, Z. Phys. Chem. Frankfurt 46, 63 [1965].

${ }^{6}$ R. Haase u. H.-J. Jansen, Z. Phys. Chem. Frankfurt 61, 310 [1968]. dadurch umgehen, daß man die ,ionischen Bestandteile" einführt ${ }^{11}$. Alle folgenden Ausführungen bleiben daher exakt, wenn man an die Stelle des Ausdrucks „Ionenart“ den allgemeineren Begriff „ionischer Bestandteil" setzt.

Die beiden Elektrolyte der Schmelze werden als Komponente 1 und Komponente 2 der binären flüssigen Mischung betrachtet. Die nur in der Komponente 1 enthaltene Ionensorte bezeichnen wir als Teilchenart $\alpha$, die lediglich aus der Komponente 2 stammende Ionensorte als Teilchenart $\beta$ und die beiden Komponenten gemeinsame Ionensorte als Teilchenart $\gamma$. Das Zeichen $z_{\mathrm{i}}(\mathrm{i}=\alpha, \beta, \gamma)$ bzw. $v_{\mathrm{i}}(\mathrm{i}=\alpha, \beta)$ bedeutet die Ladungszahl bzw. Zerfallszahl der Ionenart i. So gilt z. B. für $\mathrm{PbCl}_{2}+\mathrm{PbBr}_{2}$ :

$$
z_{\alpha}=z_{\beta}=-1, \quad z_{\gamma}=2, \quad v_{\alpha}=v_{\beta}=2 .
$$

Alle Transportgrößen, die vom Bezugssystem abhängen, werden zweckmäßigerweise relativ zur Teilchenart $\gamma$ gemessen. Die mittlere Geschwindigkeit der Ionen der Sorte $\gamma$ stellt mithin die Bezugsgeschwindigkeit dar, so daß die Ionenart $\gamma$ die Rolle

\footnotetext{
7 B. R. Sundheim u. J. D. Kellner, J. Phys. Chem. 69, 1204 [1965].

8 J. Dupuy, Y. Nakamura, S. Brillant u. J. Brenet, J. Chim. Phys. 1966, 886. - M. Bakès, S. Brillant, J. Brenet, J. Dupuy, J. Guion, Y. Nakamura u. J. Ruch, J. Chim. Phys. 1966, 1491.

9 C. Sinistri, Z. Naturforsch. 21 a, 753 [1966]. - C. SiniStri u. E. Pezzati, Ric. Sci. 37, 111 [1967]; Z. Naturforsch. 22 a, 590 [1967]. - C. Sinistri u. C. Margheritis, Z. Naturforsch. 23 a, 1155 [1968].

10 K. Wallin u. A. Lundén, Z. Naturforsch. 22 a. 591 [1967].

11 H. Schönert u. C. Sinistri, Z. Elektrochem. 66, 413 [1962].
} 
des Lösungsmittels bei Elektrolytlösungen übernimmt. So bedeutet $t_{\alpha}$ bzw. $t_{\beta}$ die Hittorfsche Überführungszahl der Ionenart $\alpha$ bzw. $\beta$ mit der Ionensorte $\gamma$ als Bezugsteilchenart. Es gilt die Identität

$$
t_{\alpha}+t_{\beta}=1 \text {. }
$$

Analog ist ${ }^{*} S_{\alpha}$ bzw. ${ }^{*} S_{\beta}$ die Überführungsentropie der Ionenart $\alpha$ bzw. $\beta$ im Hittorfschen Bezugssystem mit der Ionensorte $\gamma$ als Bezugsteilchenart. Wie wir sehen werden, können ${ }^{*} S_{\alpha}$ und ${ }^{*} S_{\beta}$ aus Messungen an Thermoketten abgeleitet werden, falls die Überführungszahlen und gewisse andere Größen bekannt sind.

Eine Thermokette der hier interessierenden Art kann durch ein Phasenchema des Typs

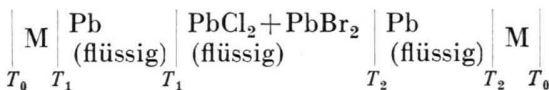

gekennzeichnet werden, worin M die Endphase (metallische Elektrodenableitung), $T_{0}$ die Zimmertemperatur und $T_{1}$ bzw. $T_{2}$ die Temperatur der linken bzw. rechten Elektrode darstellt. Meßbar sind an einer solchen nicht-isothermen galvanischen Kette insbesondere der Anfangswert $\varepsilon_{0}$ und der Endwert $\varepsilon_{\infty}$ der Thermokraft. Dabei bezieht sich $\varepsilon_{0}$ auf den Ausgangszustand einer anfangs hinsichtlich der $\mathrm{Zu}$ sammensetzung homogenen Schmelze und $\varepsilon_{\infty}$ auf den stationären Endzustand mit ungleichförmiger Zusammensetzung, der durch die Überlagerung von Diffusion und Thermodiffusion zustande kommt. Diejenige Ionenart, für welche die Elektroden der Thermokette reversibel sind, wird durch den Index r gekennzeichnet. Für das Beispiel (2) gilt demnach: $\mathbf{r}=\gamma=\mathrm{Pb}^{++}$. Wir müssen generell die drei Fälle $\mathrm{r}=\alpha, \mathrm{r}=\beta$ und $\mathrm{r}=\gamma$ unterscheiden.

SCHÖNERT und SINISTRI ${ }^{11}$ haben mit Hilfe der Methoden der Thermodynamik der irreversiblen Prozesse ${ }^{12}$ die allgemeinen Ausdrücke für $\varepsilon_{0}$ und $\varepsilon_{\infty}$ abgeleitet. Bei Berücksichtigung der hier eingeführten Definitionen und Fallunterscheidungen erhält man folgende Formeln:

$\mathrm{r}=\alpha$ :

$\mathfrak{F} \varepsilon_{0}=\frac{S_{\mathrm{m}}}{z_{\alpha}}-t_{\beta}\left(\frac{S_{1}}{z_{\alpha} v_{\alpha}}-\frac{S_{2}}{z_{\beta} v_{\beta}}\right)$

$$
-{ }^{*} S_{\Theta}-\frac{t_{\alpha}}{z_{\alpha}}{ }^{*} S_{\alpha}-\frac{t_{\beta}}{z_{\beta}}{ }^{*} S_{\beta},
$$

$\mathfrak{F} \varepsilon_{\infty}=\frac{S_{\mathrm{m}}}{z_{\alpha}}-{ }^{*} S_{\Theta}-\frac{{ }^{*} S_{\alpha}}{z_{\alpha}} ;$

12 Vgl. R. HaAse, Thermodynamik der irreversiblen Prozesse, Verlag Steinkopff, Darmstadt 1963. $\mathrm{r}=\beta:$

$\widetilde{\mho} \varepsilon_{0}=\frac{S_{\mathrm{m}}}{z_{\beta}}+t_{u}\left(\frac{S_{1}}{z_{\alpha} v_{\alpha}}-\frac{S_{2}}{z_{\beta} v_{\beta}}\right)$

$-{ }^{*} S_{\Theta}-\frac{t_{\alpha}}{z_{\alpha}}{ }^{*} S_{\alpha}-\frac{t_{\beta}}{z_{\beta}}{ }^{*} S_{\beta}$,

$\mathfrak{F} \varepsilon_{\infty}=\frac{S_{\mathrm{m}}}{z_{\beta}}-{ }^{*} S_{\Theta}-\frac{{ }^{*} S_{\beta}}{z_{\beta}} ;$

$\mathrm{r}=\gamma:$

$\mathfrak{F} \varepsilon_{0}=\frac{S_{\mathrm{m}}}{z_{\gamma}}+\frac{t_{\alpha}}{z_{\alpha} v_{\alpha}} S_{1}+\frac{t_{\beta}}{z_{\beta} v_{\beta}} S_{2}$

$-{ }^{*} S_{\Theta}-\frac{t_{\alpha}}{z_{\alpha}}{ }^{*} S_{\alpha}-\frac{t_{\beta}}{z_{\beta}}{ }^{*} S_{\beta}$,

$\widetilde{F} \varepsilon_{\infty}=\frac{S_{\mathrm{m}}}{z_{\gamma}}-{ }^{*} S_{\Theta}$

$$
+\frac{(1-x) S_{1}+x S_{2}-v_{\alpha}(1-x){ }^{*} S_{\alpha}-v_{\beta} x{ }^{*} S_{\beta}}{z_{\alpha} v_{\alpha}(1-x)+z_{\beta} v_{\beta} x} .
$$

Darin ist $\mathfrak{F}$ die Faraday-Konstante, $S_{\mathrm{m}}$ eine bestimmte Linearkombination von (partiellen) molaren Entropien der Elektrodensubstanzen - beim Beispiel (2) einfach die molare Entropie des flüssigen Bleis - , $S_{1}$ bzw. $S_{2}$ die partielle molare Entropie der Komponente 1 bzw. 2 in der Schmelze, ${ }^{*} S_{\Theta}$ die Überführungsentropie der Elektronen in der Endphase und $x$ der stöchiometrische Molenbruch der Komponente 2, durch den wir die Zusammensetzung der Elektrolytschmelze beschreiben. Bei gegebenem Druck hängen $S_{\mathrm{m}}$ und ${ }^{*} S_{\Theta}$ nur von der Temperatur $T$, die Größen $S_{1}, S_{2}, t_{\alpha}, t_{\beta},{ }^{*} S_{\alpha},{ }^{*} S_{\beta}$ aber von $T$ und $x$ ab.

Vertauscht man die Indizes 1 und 2, die Indizes $\alpha$ und $\beta$ sowie die Größen $x$ und $1-x$ miteinander, so werden die Beziehungen (3) und (4) mit den Formeln (5) und (6) identisch, während Gl. (7) und (8) erhalten bleiben. Dies muß so sein, weil die Wahl der Komponenten willkürlich ist.

Die Beziehungen (3) bis (8) sind komplizierter als die entsprechenden Formeln bei Elektrolytlösungen. Gl. (8), die nur implizit in der Arbeit von SCHÖNERT und SINISTRI ${ }^{11}$ enthalten ist, hat ein besonders verwickeltes Aussehen. Der Hintergrund für diese Formel ist die Tatsache, daß in dem Ausdruck für $\varepsilon_{\infty}$ primär stets die Überführungsentropie der Ionenart $r$ (mit einer der beiden anderen Ionensorten als Bezugsteilchenart) erscheint und daher für $\mathrm{r}=\gamma$ auf das von uns gewählte Bezugssystem (Bezugsteilchenart $\gamma$ ) umgerechnet werden muß.

Lösen wir die Gln. (3) und (4) oder (5) und (6) oder (7) und (8) nach ${ }^{*} S_{a}$ und ${ }^{*} S_{\beta}$ auf, so erkennen wir, daß auf der rechten Seite bekannte oder meßbare Größen stehen. Es können also hier, genau 
wie bei Elektrolytlösungen mit zwei Ionensorten, die Überführungsentropien zweier Ionenarten bei jeder Temperatur und Zusammensetzung experimentell bestimmt werden. Eine Aufspaltung von ${ }^{*} S_{\mathrm{i}}$ in zwei Anteile nach der Beziehung

$$
{ }^{*} S_{\mathrm{i}}={ }^{*} Q_{\mathrm{i}} / T+S_{\mathrm{i}} \quad(\mathrm{i}=\alpha, \beta),
$$

worin ${ }^{*} Q_{\mathrm{i}}$ bzw. $S_{\mathrm{i}}$ die Überführungswärme bzw. partielle molare Entropie der Ionenart i bedeutet, ist vom makroskopischen Standpunkt willkürlich ${ }^{12}$. In der angelsächsischen Literatur wird meist ${ }^{*} Q_{\mathrm{i}} / T$ als "entropy of transport" oder "entropy of transfer" und " $S_{\mathrm{i}}$ als "transported entropy" bezeichnet.

\section{Formeln für reine Schmelzen}

Wir betrachten nun die Grenzfälle $x=0$ (reine flüssige Komponente 1, Ionenarten $\alpha$ und $\gamma$ ) und $x=1$ (reine flüssige Komponente 2, Ionenarten $\beta$ und $\gamma)$. Wir bezeichnen mit $S_{01}$ bzw. $S_{02}$ die molare Entropie des reinen flüssigen Elektrolyten 1 bzw. 2 und mit ${ }_{0}^{*} S_{\alpha}$ bzw. ${ }_{0}^{*} S_{\beta}$ die Überführungsentropie der Ionenart $\alpha$ bzw. $\beta$ für $x=0$ bzw. $x=1$. Wir berücksichtigen ferner die Definition von $t_{\alpha}$ bzw. $t_{\beta}$ und beachten Gl. (1). Wir finden dann:

$x=0: \quad t_{\alpha}=1, \quad t_{\beta}=0, \quad S_{1}=S_{01}, \quad{ }^{*} S_{\alpha}={ }_{0}^{*} S_{\alpha} ;$

$x=1: \quad t_{u}=0, \quad t_{\beta}=1, \quad S_{2}=S_{02}, \quad{ }^{*} S_{\beta}={ }_{0}^{*} S_{\beta}$.

Bei gegebenem Druck hängen $S_{01}, S_{02},{ }_{0}^{*} S_{\alpha}$ und ${ }_{0}^{*} S_{\beta}$ ausschließlich von der Temperatur ab. Mit dem Grenzverhalten von ${ }^{*} S_{\alpha}$ für $x \rightarrow 1$ und von ${ }^{*} S_{\beta}$ für $x \rightarrow 0$ beschäftigen wir uns später.

Es sei $\varepsilon_{\mathrm{I}}$ bzw. $\varepsilon_{\mathrm{II}}$ die Thermokraft einer Thermokette für $x=0$ bzw. $x=1$, also einer Kette des Typs [vgl. (2) ]

$$
\left.\mathrm{Pb} \underset{T_{1}}{\mid} \mathrm{PbCl}_{2}\right|_{T_{2}} \mathrm{~Pb}
$$

bzw.

$$
\left.\mathrm{Pb} \underset{T_{1}}{\mid} \mathrm{PbBr}_{2}\right|_{T_{2}} \mathrm{~Pb}
$$

mit $\mathrm{PbCl}_{2}$ bzw. $\mathrm{PbBr}_{2}$ als Komponente 1 bzw. 2. In einer solchen Thermokette gibt es keine Konzentrationsverschiebung. Deshalb entspricht $\varepsilon_{\mathrm{I}}$ bzw. $\varepsilon_{\text {II }}$ dem Grenzwert sowohl von $\varepsilon_{0}$ als auch von $\varepsilon_{\infty}$ für $x=0$ bzw. $x=1$. Da für $x=0$ nur die Fälle $\mathrm{r}=\alpha$, $\mathrm{r}=\gamma$ und für $x=1$ lediglich die Fälle $\mathrm{r}=\beta, \mathrm{r}=\gamma$ in Frage kommen, leiten wir aus Gl. (3) bis (9) ab:

$\mathrm{r}=\alpha$ :

$\widetilde{\mho} \varepsilon_{\mathrm{I}}=\frac{S_{\mathrm{nn}}}{z_{\alpha}}-{ }^{*} S_{\Theta}-\frac{{ }_{0}^{*} S_{\alpha}}{z_{\alpha}} ;$ $\mathrm{r}=\beta$ :

$\widetilde{F} \varepsilon_{\mathrm{II}}=\frac{S_{\mathrm{m}}}{z_{\beta}}-{ }^{*} S_{\Theta}-\frac{{ }_{0}^{*} S_{\beta}}{z_{\beta}} ;$

$\mathrm{r}=\gamma^{\prime}:$

$\widetilde{F} \varepsilon_{\mathrm{I}}=\frac{S_{\mathrm{m}}}{z_{\gamma}}+\frac{S_{01}}{z_{\alpha} v_{\alpha}}-{ }^{*} S_{\Theta}-\frac{{ }_{0}^{*} S_{\alpha}}{z_{\alpha}} ;$

$\mathfrak{F} \varepsilon_{\mathrm{II}}=\frac{S_{\mathrm{m}}}{z_{\gamma}}+\frac{S_{02}}{z_{\beta} \nu_{\beta}}-{ }^{*} S_{\Theta}-\frac{{ }_{0}^{*} S_{\beta}}{z_{\beta}}$.

Man kann nun z. B. die Thermokette (10) einerseits als Grenzfall von (2), andererseits als Grenzfall von

$$
\mathrm{Pb} \underset{T_{1}}{\mid} \mathrm{PbCl}_{2}+\left.\mathrm{NaCl}\right|_{T_{2}} \mathrm{~Pb}
$$

mit $\mathrm{PbCl}_{2}$ bzw. $\mathrm{NaCl}$ als Komponente 1 bzw. 2 auffassen. Im ersten Falle gilt Gl. (14) mit $\alpha=\mathrm{Cl}^{-}$, $\gamma=\mathrm{Pb}^{++}$, im zweiten Falle Gl. (12) mit $\alpha=\mathrm{Pb}^{++}$, $\gamma=\mathrm{Cl}^{-}$. Da beide Gleichungen sich auf dasselbe System beziehen, müssen die Ausdrücke (12) und (14) daselbe Resultat liefern. Dies wiederum bedeutet, daß die Beziehung

$$
{ }_{0}^{*} S_{\mathrm{Pb}}{ }^{++}+2{ }_{0}^{*} S_{\mathrm{Cl}^{-}}=S_{01}
$$

bestehen muß.

Bei beliebigen reinen Schmelzen mit zwei Ionenarten bezeichnen wir die Zerfallszahl der Kationen bzw. Anionen mit $v_{+}$bzw. $v_{-}$und die Überführungsentropie der Kationen bzw. Anionen mit ${ }^{*} S_{+}$ bzw. ${ }^{*} S_{-}$. (Für ${ }^{*} S_{+}$sind die Anionen, für ${ }^{*} S_{-}$die Kationen die Bezugsteilchen.) Ferner schreiben wir statt $S_{01}$ oder $S_{02}$ einfach $\bar{S}$. Dann folgt aus (12) und (14) oder (13) und (15):

$$
v_{+}{ }^{*} S_{+}+v_{-}{ }^{*} S_{-}=\bar{S},
$$

die Verallgemeinerung von Gl. (17). Es gibt also hier, wie zu erwarten, nur eine einzige unabhängige Überführungsentropie.

Die Formeln (12) bis (15) und (18) stimmen mit den auf direktem Wege abgeleiteten Beziehungen ${ }^{13}$ für reine flüssige Elektrolyte völlig überein. Es sei als historische Kuriosität angemerkt, daß die ersten Thermoketten, die überhaupt untersucht wurden, nicht etwa Elektrolytlösungen, sondern reine Salzschmelzen enthielten (ANDREws 1837).

\section{Auswertung von Literaturdaten}

Bei reinen Elektrolytschmelzen läßt sich die Überführungsentropie ${ }^{*} S_{+}$bzw. ${ }^{*} S_{-}$relativ leicht aus

13 R. HAASE ${ }^{12}$, S. 437 bis S. 439 und S. 449. 
experimentellen Daten ableiten, weil neben der zu messenden Thermokraft gemäß Gl. (12) bis (15) nur molare Entropien reiner Stoffe und Elektronenüberführungsentropien bekannt zu sein brauchen. Beispiele für so ermittelte Überführungsentropien finden sich bereits in der Literatur ${ }^{13-17}$. Nach den bisherigen Untersuchungen nimmt die positive Größe ${ }^{*} S_{+}$bzw. ${ }^{*} S_{-}$mit steigender Temperatur stets zu.

Die Auswertung der Messungen an binären Elektrolytschmelzen erfordert, wie aus Gl. (3) bis (8) hervorgeht, erheblich mehr Aufwand als bei reinen Schmelzen. Einerseits müssen hier Meßwerte sowohl von $\varepsilon_{0}$ als auch von $\varepsilon_{\infty}$ vorliegen. Andererseits müssen die Überführungszahlen und die partiellen molaren Entropien der beiden Komponenten in der Schmelze bekannt sein. Es ist daher nicht verwunderlich, daß es noch keine vollständigen Angaben über die beiden unabhängigen Überführungsentropien einer binären Schmelze gibt.

Das einzige binäre System, für das eine Berechnung von Überführungsentropien aus Literaturdaten möglich erscheint, ist die Salzschmelze $\mathrm{NaNO}_{3}+\mathrm{AgNO}_{3}$ bei $310^{\circ} \mathrm{C}$. Sundheim und KelL$\mathrm{NER}^{7}$ haben nämlich Meßwerte von $\varepsilon_{0}$ und $\varepsilon_{\infty}$ an der Thermokette

$$
\begin{array}{l|l|l}
\mathrm{Ag} & \begin{array}{l}
\mathrm{NaNO}_{3}+\mathrm{AgNO}_{3} \\
\text { (fest) }
\end{array} & \begin{array}{l}
\mathrm{Ag} \\
T_{1}
\end{array} \\
\text { (fest) }
\end{array}
$$

für mehrere Zusammensetzungen der Schmelze bei einer mittleren Temperatur von $310^{\circ} \mathrm{C}$ mitgeteilt ${ }^{18}$.

Wir fassen $\mathrm{NaNO}_{3}$ bzw. $\mathrm{AgNO}_{3}$ als Komponente 1 bzw. 2 der binären Schmelze auf, deren Zusammensetzung wir durch den Molenbruch $x$ des Silbernitrats kennzeichnen. Ist $\bar{S}_{\mathrm{Ag}}$ die molare Entropie des festen Silbers bei $310^{\circ} \mathrm{C}$, so gelten für die Kette (19) die Formeln (5) und (6) mit

$$
\begin{gathered}
S_{\mathrm{m}}=\bar{S}_{\mathrm{Ag}}, \quad z_{\alpha}=z_{\beta}=v_{\alpha}=v_{\beta}=1, \\
t_{\alpha}=t_{\mathrm{Na}}{ }^{+}, \quad t_{\beta}=t_{\mathrm{Ag}^{+}}=1-t_{\mathrm{Na}^{+}}, \\
{ }^{*} S_{\alpha}={ }^{*} S_{\mathrm{Na}^{+}}, \quad{ }^{*} S_{\beta}={ }^{*} S_{\mathrm{Ag}^{+}} .
\end{gathered}
$$

Da die Endphase bei der Kette (19) ebenfalls Silber ist, bedeutet ${ }^{*} S_{\Theta}$ in Gl. (5) und (6) die Elektronenüberführungsentropie im festen Silber bei $310^{\circ} \mathrm{C}$.

14 K. S. Pitzer, J. Phys. Chem. 65, 147 [1961].

15 C. Sinistri, Z. Naturforsch. 20 a, 1045 [1965].

16 A. Kvist u. A. Randsalu, Z. Naturforsch. 21 a, 278 [1966].

17 W. Fischer, Z. Naturforsch. 21 a, 281 [1966].

18 Auch andere Autoren haben $\varepsilon_{0}$ an dieser Kette experimentell bestimmt. Da aber SundHeim und Kellner ${ }^{7}$ die einzigen Autoren sind, die für eine Thermokette mit Elektrolytschmelzen den Zeiteffekt und damit $\varepsilon_{\infty}$ gemessen haben,
Die Größen $\bar{S}_{\mathrm{Ag}}$ und ${ }^{*} S_{\Theta}$ sind bekannt, so daß nach Gl. (6) und (20) aus den $\varepsilon_{\infty}$-Messungen die Überführungsentropie ${ }^{*} S_{\mathrm{Ag}^{+}}$der Silberionen in $\mathrm{Ab}$ hängigkeit von $x$ bei $310{ }^{\circ} \mathrm{C}$ sofort gewonnen werden kann. Wesentlich schwieriger ist die Ermittlung der Überführungsentropie ${ }^{*} S_{\mathrm{Na}^{+}}$der Natriumionen gemäß Gl. (5) und (20) aus den $\varepsilon_{0}$-Messungen, weil wir die Überführungszahl $t_{\mathrm{Na}^{+}}\left(=1-t_{\mathrm{Ag}^{+}}\right)$und die partielle molare Entropie $S_{1}$ bzw. $S_{2}$ des Natriumnitrats bzw. Silbernitrats benötigen. In der Tat ist von den genannten Autoren ${ }^{7}$ nur ${ }^{*} S_{\mathrm{Ag}^{+}}$, nicht aber ${ }^{*} S_{\mathrm{Na}}{ }^{+}$berechnet worden. Wir wollen daher versuchen, annähernde Werte für ${ }^{*} S_{\mathrm{Na}^{+}}$aus Literaturdaten abzuleiten.

Überführungsmessungen nach der Hittorfschen Methode ergeben für $\mathrm{NaNO}_{3}+\mathrm{AgNO}_{3}$ bei $305{ }^{\circ} \mathrm{C}$ die einfachen Relationen ${ }^{19}$

$$
t_{\mathrm{Na}}{ }^{+}=1-x, \quad t_{\mathrm{Ag}^{+}}=x,
$$

deren Gültigkeit bei $310^{\circ} \mathrm{C}$ wir ohne weiteres annehmen dürfen ${ }^{20}$.

Weiterhin bekannt sind EMK-Werte ${ }^{21}$ für eine chemische Kette (d. h. eine reversible Kette ohne Temperatur-, Druck- und Konzentrationsgefälle) mit einer für $\mathrm{NO}_{3}{ }^{-}$reversiblen Elektrode:

$$
\begin{array}{l|l|l}
\mathrm{Ag} & \begin{array}{l}
\mathrm{NaNO}_{3}+\mathrm{AgNO}_{3} \\
\text { (flüssig) }
\end{array} & \mathrm{O}_{2}, \mathrm{NO}_{2}(\mathrm{Pt}) .
\end{array}
$$

Kalorimetrische Daten für die Mischungswärmen bei der Salzschmelze $\mathrm{NaNO}_{3}+\mathrm{AgNO}_{3}$ sind ebenfalls vorhanden. Man gelangt so zu den thermodynamischen Mischungsgrößen für $\mathrm{NaNO}_{3}+\mathrm{AgNO}_{3}$ in Abhängigkeit von der Zusammensetzung bei der jeweils vorgegebenen Temperatur. Auf diese Weise läßt sich zeigen ${ }^{21}$, daß die partiellen molaren Entropien $S_{1}$ und $S_{2}$ folgende Gestalt haben:

$$
\begin{aligned}
& S_{1}=S_{01}-R \ln (1-x)+B x^{2}, \\
& S_{2}=S_{02}-R \ln x+B(1-x)^{2} .
\end{aligned}
$$

Darin ist $R$ die Gaskonstante und $B$ eine prinzipiell temperaturabhängige Größe, deren $\operatorname{Wert}^{21}$ bei $350{ }^{\circ} \mathrm{C}$

$$
B=-0,64 \mathrm{cal} \mathrm{K}^{-1} \mathrm{~mol}^{-1}
$$

beschränken wir die Diskussion auf die Daten dieser Autoren.

19 F. R. Duke, R.W. Laity u. B. Owens, J. Electrochem. Soc. 104, 299 [1957].

20 Die interessanten Konsequenzen des Ansatzes (21) werden im Anhang besprochen.

21 J. A. A. Ketelaar u. A. Dammers-De Klerk, Koninkl. Ned. Akad. Wetenschap. Proc. B 68, 169 [1965]. 
innerhalb der Fehlergrenzen der verschiedenen Messungen auch für $310{ }^{\circ} \mathrm{C}$ richtig sein dürfte. (Das Symbol cal bezeichnet die thermochemische Kalorie, definiert durch $\mathrm{cal}=4,184 \mathrm{~J}$.)

Aus Gln. (5), (6), (20), (21) und (23) finden wir:

$$
\begin{gathered}
{ }^{*} S_{\mathrm{Na}}{ }^{+}=\frac{{ }^{*} S_{\mathrm{Ag}^{+}}=\bar{S}_{\mathrm{Ag}}-{ }^{*}{ }^{*} S_{\Theta}-\widetilde{F} \varepsilon_{0}-\widetilde{F} \varepsilon_{\infty},}{1-x}+S_{01}-S_{02}+R \ln \frac{x}{1-x} \\
+B(2 x-1)-\frac{x}{1-x}{ }^{*} S_{\mathrm{Ag}}{ }^{+} .
\end{gathered}
$$

Beachten wir Gl. (24) sowie die Literaturwerte ${ }^{7,22}$ für $310^{\circ} \mathrm{C}$

$$
\begin{aligned}
{\overline{S_{\mathrm{Ag}}}}= & 14,35 \mathrm{cal} \mathrm{K}^{-1} \mathrm{~mol}^{-1}, \quad{ }^{*} S_{\Theta} \approx 0, \\
& S_{01}=52,8 \mathrm{cal} \mathrm{K}^{-1} \mathrm{~mol}^{-1}, \\
& S_{02}=58,7 \mathrm{cal} \mathrm{K}^{-1} \mathrm{~mol}^{-1}
\end{aligned}
$$

und die Werte der universellen Konstanten

$$
\begin{aligned}
& \widetilde{V}=23062 \mathrm{cal} \mathrm{V}^{-1} \mathrm{~mol}^{-1}, \\
& R=1,9872 \mathrm{cal} \mathrm{K}^{-1} \mathrm{~mol}^{-1},
\end{aligned}
$$

\begin{tabular}{|c|c|c|c|c|}
\hline$x$ & $\frac{-\varepsilon_{0}}{\mathrm{VK} \mathrm{K}^{-1}} \cdot 10^{6}$ & $\frac{-\varepsilon_{\infty}}{\mathrm{VK}^{-1}} \cdot 10^{6}$ & $\frac{* S_{\mathrm{Na}+}}{\text { cal } \mathrm{K}^{-1} \mathrm{~mol}^{-1}}$ & $\frac{* S_{\mathrm{Ag}+}}{\text { cal K }{ }^{-1} \mathrm{~mol}^{-1}}$ \\
\hline 0,05 & 419 & 149 & 13,2 & 17,8 \\
\hline 0,20 & 345 & 215 & 14,8 & 19,3 \\
\hline 0,35 & 338 & 242 & 16,4 & 19,9 \\
\hline 0,50 & 331 & 248 & 18,0 & 20,0 \\
\hline 0,60 & 327 & 239 & 19,7 & 19,9 \\
\hline 0,75 & 322 & 205 & 25,8 & 19,1 \\
\hline 0,90 & 312 & 156 & 51,9 & 18,0 \\
\hline 1,00 & $\begin{array}{c}319 \\
\left(\varepsilon_{0}=\varepsilon_{\mathrm{II}}\right)\end{array}$ & - & - & $\left(* S_{\mathrm{Ag}}^{+21,7}={ }_{0}^{*} S_{\mathrm{Ag}^{+}}\right)$ \\
\hline
\end{tabular}

so erhalten wir aus den $\varepsilon_{0}$ - und $\varepsilon_{\infty}$-Messungen nach Gl. (25) und (26) die Funktionen ${ }^{*} S_{\mathrm{Na}^{+}}+(x)$ und ${ }^{*} S_{\mathrm{Ag}^{+}}(x)$ für $\mathrm{NaNO}_{3}+\mathrm{AgNO}_{3}$ bei $310{ }^{\circ} \mathrm{C}$.

In Tab. 1 sind die experimentellen Werte ${ }^{7}$ von $\varepsilon_{0}$ und $\varepsilon_{\infty}$ sowie die daraus von uns berechneten Größen ${ }^{*} S_{\mathrm{Na}^{+}}$und ${ }^{*} S_{\mathrm{Ag}^{+}}$zusammengestellt. Abb. 1 zeigt die Funktionen ${ }^{*} S_{\mathrm{Na}^{+}}(x)$ und ${ }^{*} S_{\mathrm{Ag}^{+}}(x)$.

Tab. 1. Anfangswert $\varepsilon_{0}$ und Endwert $\varepsilon_{\infty}$ der Thermokraft der Thermokette (19) sowie Überführungsentropie ${ }^{*} S_{\mathrm{Na}^{+}}$der Natriumionen und Überführungsentropie ${ }^{*} S_{\mathrm{Ag}}$ der Silberionen in Abhängigkeit vom Molenbruch $x$ des Silbernitrats für die Salzschmelze $\mathrm{NaNO}_{3}+\mathrm{AgNO}_{3}$ bei $310{ }^{\circ} \mathrm{C}$.

Der in Tab. 1 für $x=1$ (reines Silbernitrat) angegebene Wert von $\varepsilon_{0}$ ist die Thermokraft $\varepsilon_{\text {II }}$ der Thermokette

$$
\mathrm{Ag}{ }_{T_{1}} \mathrm{AgNO}_{3} T_{T_{2}} \mathrm{Ag}
$$

22 K. K. Kelley, U.S. Dept. Interior, Bur. Mines, Bull. 371 [1934], 477 [1948], 584 [1960]. bei $310{ }^{\circ} \mathrm{C}$. Eigentlich müßten die auf $x=1$ extrapolierten Werte von $\varepsilon_{0}$ und $\varepsilon_{\infty}$ mit $\varepsilon_{\text {II }}$ übereinstimmen. Da dies nach Tab. 1 , besonders in bezug auf $\varepsilon_{\infty}$, nicht zutrifft, sind die Messungen in diesem Bereich nicht konsistent.

Die Zahlenangaben für ${ }^{*} S_{\mathrm{Ag}^{+}}$in Tab. 1 und Abb. 1 stimmen im wesentlichen mit denen von SUNDHEIM und KeLLnER ${ }^{7}$ überein. Die Überführungsentropie ${ }_{0}^{*} S_{\mathrm{Ag}^{+}}$der Silberionen im reinen flüssigen Silbernitrat

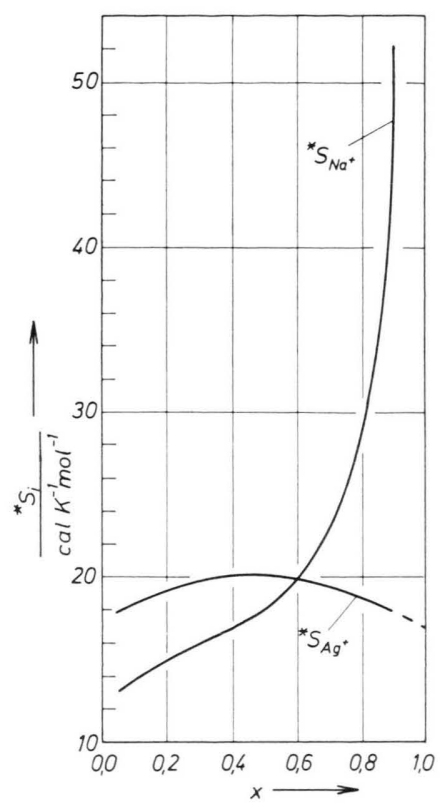

Abb. 1. System $\mathrm{NaNO}_{3}+\mathrm{AgNO}_{3}$ bei $310{ }^{\circ} \mathrm{C}$ : Überführungsentropie ${ }^{*} S_{\mathrm{Na}^{+}}$der Natriumionen und Überführungsentropie ${ }^{*} S_{\mathrm{Ag}}$ der Silberionen in Abhängigkeit vom Molenbruch $x$ des Silbernitrats.

(s. Tab. 1) ist nach Gl. (13), (20), (27) und (28) berechnet worden. Dieser Wert ${ }_{0}^{*} S_{\mathrm{Ag}^{+}} \approx 22 \mathrm{cal} \mathrm{K}{ }^{-1}$ $\mathrm{mol}^{-1}$ ) weicht nicht wesentlich von den Daten anderer Autoren ${ }^{13-15}$ ab. Graphische Extrapolation (s. Abb. 1) ergibt allerdings ${ }_{0}^{*} S_{\mathrm{Ag}^{+}} \approx 17 \mathrm{cal} \mathrm{K}^{-1} \mathrm{~mol}^{-1}$. Dies hängt mit den erwähnten Inkonsistenzen zusammen. Der ebenfalls durch graphische Extrapolation ermittelbare Wert der Überführungsentropie ${ }_{0}^{*} S_{\mathrm{Na}^{+}}$der Natriumionen im reinen flüssigen Natriumnitrat $\left({ }_{0}^{*} S_{\mathrm{Na}^{+}} \approx 12 \mathrm{cal} \mathrm{K}^{-1} \mathrm{~mol}^{-1}\right)$ kann z. Z. noch nicht durch direkte Messungen bestätigt werden.

Offen bleibt auch das Grenzverhalten von ${ }^{*} S_{\mathrm{Na}{ }^{+}}$ bei $x \rightarrow \mathrm{l}$, $\mathrm{d}$. h. bei verschwindender Natriumnitratkonzentration, und von ${ }^{*} S_{\mathrm{Ag}^{+}}$bei $x \rightarrow 0$, d. h. bei verschwindender Silbernitratkonzentration. Derartige Grenzwerte können prinzipiell verschwinden, unendlich werden oder endlich und von Null ver- 
schieden sein. Wir wagen es nicht, aus Abb. 1 irgendwelche Schlüsse zu ziehen, weil die beiden Kurven zu unterschiedlichen Verlauf zeigen und genaue Meßwerte bei hohen Verdünnungen sowie entsprechende Daten für andere Systeme fehlen.

\section{Anhang}

Wir wollen die Konsequenzen des Ansatzes (21), der - wenigstetns näherungsweise - für das flüssige $S y$ stem $\mathrm{NaNO}_{3}+\mathrm{AgNO}_{3}$ zu gelten scheint, kurz untersuchen. Dabei setzen wir einige Grundformeln, deren Ableitung an anderer Stelle ${ }^{11,23}$ nachzulesen ist, als bekannt voraus. (21)

Wir nehmen an, es gelte die Verallgemeinerung von

$$
t_{\alpha}=1-x, \quad t_{\beta}=x
$$

für eine Schmelze, die aus zwei 1-1-wertigen Elektrolyten mit gemeinsamen Anionen (wie $\mathrm{NaNO}_{3}+\mathrm{AgNO}_{3}$ ) besteht. Wir haben also die Bedingungen

$$
z_{\alpha}=z_{\beta}=1, \quad z_{\gamma}=-1, \quad v_{\alpha}=v_{\beta}=1,
$$

die unsere Rechnungen weitgehend vereinfachen.

Für die Ionenleitfähigkeiten $\lambda_{a}$ und $\lambda_{\beta}$ (gemessen relativ zur gemeinsamen Ionenart $\gamma$ ) ergibt sich zunächst allgemein :

$$
\lambda_{\alpha}=\frac{t_{\alpha} \varkappa \bar{V}}{\left|z_{\alpha}\right| v_{\alpha}(1-x)}, \quad \lambda_{\beta}=\frac{t_{\beta} \varkappa \bar{V}}{\left|z_{\beta}\right| v_{\beta} x} .
$$

Darin sind $\varkappa$ und $\bar{V}$ die (spezifische) Leitfähigkeit und das molare Volumen der Schmelze. Mit Hilfe von Gl. (1) folgt aus Gl. (32) :

$$
\left|z_{\alpha}\right| v_{\alpha}(1-x) \lambda_{\alpha}+\left|z_{\beta}\right| v_{\beta} x \lambda_{\beta}=\varkappa \bar{V} .
$$

Führen wir die Voraussetzungen (30) und (31) in (32) ein, so finden wir:

$$
\lambda_{\alpha}=\lambda_{\beta}=\varkappa \bar{V},
$$

während Gl. (33) zu einer Identität wird. Die Ionenleitfähigkeiten (und damit die Beweglichkeiten) der beiden Ionenarten $\alpha$ und $\beta$ werden mithin einander gleich, und zwar bei jeder beliebigen Zusammensetzung der Schmelze.

Wir betrachten jetzt eine Konzentrationskette mit Überführung vom Typ

$$
\mathrm{Ag}\left|\mathrm{NaNO}_{3}+\mathrm{AgNO}_{3}\left(x_{\mathrm{I}}\right) \| \mathrm{NaNO}_{3}+\mathrm{AgNO}_{3}\left(x_{\mathrm{II}}\right)\right| \mathrm{Ag}
$$

oder

$$
\begin{aligned}
\mathrm{O}_{2}, \mathrm{NO}_{2}(\mathrm{Pt})\left|\mathrm{NaNO}_{3}+\mathrm{AgNO}_{3}\left(x_{\mathrm{I}}\right)\right| & \cdot\left|\mathrm{NaNO}_{3}+\mathrm{AgNO}_{3}\left(x_{\mathrm{II}}\right)\right| \mathrm{O}_{2}, \mathrm{NO}_{2}(\mathrm{Pt})
\end{aligned}
$$

mit zwei verschiedenen Zusammensetzungen der Schmelze, beschrieben durch die beiden Werte $x_{\mathrm{I}}$ und $x_{\mathrm{II}}$ des Molenbruches $x$ der Komponente $2\left(\mathrm{AgNO}_{3}\right)$. Die allge-

${ }^{23}$ J. Richter, Ber. Bunsenges. Phys. Chem. 72, 681 [1968]. meinen Formeln für die EMK $(\Phi)$ einer solchen isotherm-isobaren Kette lauten zunächst, wenn wir mit $a_{1}$ bzw. $a_{2}$ die Aktivität der Komponente 1 bzw. 2 bezeichnen:

$r=\alpha:$

$\mathfrak{F} \Phi=R T \int_{x_{\mathrm{I}}}^{x_{11}} \frac{z_{\alpha} v_{\alpha}(1-x)+z_{\beta} v_{\beta} x}{z_{\alpha} z_{\beta} v_{\alpha} v_{\beta} x} t_{\beta} \mathrm{d} \ln a_{1}$,

$\mathrm{r}=\beta$ :

$\mathfrak{F} \Phi=R T \int_{x_{\mathrm{I}}}^{x_{1 \mathrm{I}}} \frac{z_{\alpha} v_{\alpha}(1-x)+z_{\beta} v_{\beta} x}{z_{\alpha} z_{\beta} v_{\alpha} v_{\beta}(1-x)} t_{\alpha} \mathrm{d} \ln a_{2}$,

$\mathrm{r}=\gamma:$

$$
\begin{aligned}
\mathfrak{F} \Phi=R T\left[\frac{1}{z_{\alpha} v_{\alpha}}\right. & \ln \frac{a_{1}^{\mathrm{I}}}{a_{1} \mathrm{II}} \\
& \left.+\int_{x_{\mathrm{I}}}^{x_{\mathrm{II}}} \frac{z_{\alpha} v_{\alpha}(1-x)+z_{\beta} v_{\beta} x}{z_{\alpha} z_{\beta} v_{\alpha} v_{\beta} x} t_{\beta} \mathrm{d} \ln a_{1}\right] .
\end{aligned}
$$

$a_{k}^{\mathrm{I}}$ bzw. $a_{k}^{\mathrm{II}}(k=1,2)$ ist der Wert der Aktivität $a_{k}$ der Komponente $k$ bei der Zusammensetzung $x_{\mathrm{I}}$ bzw. $x_{\mathrm{II}}$. Berücksichtigen wir wiederum unsere Voraussetzungen (30) und (31), so gelangen wir zu folgenden einfachen Ausdrücken :

$$
\begin{array}{rlrl}
\mathrm{r}=\alpha: & \mathfrak{F} \Phi=R T \ln \left(a_{1}{ }^{\mathrm{I}} / a_{1}{ }^{\mathrm{I}}\right), \\
\mathrm{r}=\beta: & \mathfrak{F} \Phi=R T \ln \left(a_{2}{ }^{\mathrm{I}} / a_{2}{ }^{\mathrm{I}}\right), \\
\mathrm{r}=\gamma: & \Phi & =0,
\end{array}
$$

wobei das letzte Resultat besonders interessant ist. Für die Kette (35) gilt Gl. (41), für die Kette (36) aber Gl. (42). Von einer „Vernachlässigbarkeit des Diffusionspotentials“, wie man häufig in der Literatur liest, kann in keinem Falle die Rede sein. Es handelt sich vielmehr um Kompensationseffekte. Dies erkennt man besonders deutlich an der Kette (36), wo sich nach Gl. (42) Diffusions- und Elektrodenpotentiale zu Null ergänzen.

Wir behandeln schließlich eine Thermokette des Typs $\mathrm{O}_{2}, \mathrm{NO}_{2}(\mathrm{Pt}){ }_{T_{1}}\left|\mathrm{NaNO}_{3}+\mathrm{AgNO}_{3}\right| \mathrm{O}_{2}, \mathrm{NO}_{2}(\mathrm{Pt})$. (43)

Für diese gelten zunächst Gl. (7) und Gl. (8). Unter den Voraussetzungen (30) und (31) finden wir:

$$
\begin{aligned}
\mathfrak{F} \varepsilon_{0}=\mathfrak{F} \varepsilon_{\infty}= & -S_{\mathrm{m}}-{ }^{*} S_{\Theta}+(1-x) S_{1} \\
& +x S_{2}-(1-x){ }^{*} S_{a}-x{ }^{*} S_{\beta} .
\end{aligned}
$$

Der Anfangswert $\varepsilon_{0}$ und der Endwert $\varepsilon_{\infty}$ der Thermokraft sind also einander gleich. Dieses Ergebnis stimmt vollkommen mit der Aussage (42) überein, weil der Unterschied zwischen $\varepsilon_{0}$ und $\varepsilon_{\infty}$ verschwindet, wenn die EMK der zugehörigen isothermen Konzentrationskette Null wird ${ }^{12}$. Nach Gl. (44) kann man aus Messungen an der Thermokette (43) nur die Linearkombination $(1-x){ }^{*} S_{\alpha}+x{ }^{*} S_{\beta}$ der Überführungsentropien gewinnen. 